\title{
Applying the 3C Model to FLOSS Communities
}

\author{
Sara S. Fernandes ${ }^{1}$ and Luis Soares Barbosa ${ }^{2}$ \\ HASLab INESC TEC \& UNU-EGOV, United Nations University \\ Campus de Couros, Guimarães, Portugal \\ sarasantos.fernandes@gmail.com, lsb@di.uminho.pt
}

\begin{abstract}
How learning occurs within Free/Libre Open Source (FLOSS) communities and what is the dynamics such projects (e.g. the life cycle of such projects) are very relevant questions when considering the use of FLOSS projects in a formal education setting. This paper introduces an approach based on the 3C collaboration model (communication, coordination and cooperation) to represent the collaborative learning dynamics within FLOSS communities. To explore the collaborative learning potential of FLOSS communities a number of questionnaires and interviews to selected FLOSS contributors were run. From this study a $3 \mathrm{C}$ collaborative model applicable to FLOSS communities was designed and discussed.
\end{abstract}

Keywords: 3C collaboration model, FLOSS, learning

\section{Introduction}

In recent years, open software development has become more and more prevalent. With the rise and generalisation of the Internet, communities and individuals worldwide interconnect themselves and cooperate in a variety of ways. Examples range from crowd funding platforms, such as FundedByMe in Sweden or Seedrs in UK, to PulsePoint Respond which is an enterprise-class, software-as-a-service (SaaS) pre-arrival solution designed to support public safety agencies working to improve cardiac survival rates through improved bystander performance and active citizenship. And, of course, Wikipedia, probably the most used collaboration platform in the world.

Open development is essentially a collaborative process, most commonly focused on a product or service whose added value is perceived by heterogeneous communities. Participants may work in different environments, have totally different backgrounds and resources, and act under different conditions [3]. Collaboration in this sense may be seen as the combination of communication, coordination and cooperation. The articulation of these attributes is what remains in the origin of the $3 C$ collaboration model, originally proposed by Ellis et al [7] and refined in a later work [6]. Communication is related to the exchange of messages and information among people; coordination concerns management of people and comunities, their activities and resources; and finally cooperation denotes a shared activity taking place on a shared space. This model appears frequently in the literature as a means to classify collaborative systems $[11,19]$. 
The development of Free/Libre Open Source Software (FLOSS) projects across different people and communities, can be regarded as an example of a collaborative and participatory platform, maintained, and to a certain extent owned by a community. Actually, FLOSS communities consist of heterogeneous groups of independent volunteers, who interact even if driven by utterly different motivations $[4,17]$. Moreover, FLOSS development provides an example of Peer-Production [14], based as it is on collaborative, social modes of interaction and knowledge exchange [2].

In such a setting, the goal of this paper is to explore the application of the $3 \mathrm{C}$ collaboration model to FLOSS communities, under the broad objective of boosting their potential as non standard educational agents. This entails the need for a accurate understanding of what are the pillars and the dynamics underpinning FLOSS communities. Therefore, we ran an international questionnaire and performed 4 individual written interviews with active members os such communities in an attempt to understand how learning processes occur within FLOSS communities.

The rest of the paper is structured as follows. After some review of background concepts in Section 2, Section 3 presents the research method. Data gathered through a questionnaire, following up our previous research $[8,9]$, and a qualitative analysis of the interviews, is presented in section 4 . Section 5 presents the $3 \mathrm{C}$ model applied to FLOSS Communities. Finally, Section 6 concludes and presents some directions for future work.

\section{Background}

Learning can be defined as a persisting change in human performance or performance potential which must come about as a result of the learners experience and interaction with the world [5]. It can be formal, i.e. institutionally framed and hierarchically structured, or informal. Informal learning is a life-long process in which an individual acquires knowledge, attitudes, values and skills while performing daily activity within various contexts. From Jay Cross perspective, people informally acquire much of the knowledge they use in their practice. Through the observation of others, by trial and error, and simply working side by side with more experienced people. In his opinion, formal education contributes only about $10 \%$ to $20 \%$ of what a person learns in a professional context [1]. In both settings, the qualifier collaborative refers to sets of activities involving a group of people learning or trying to learn something together.

When several people learn, or attempt to learn, something together, we refer to this activity as collaborative learning. Unlike individual learning, collaborative learning capitalizes on other persons resources and skills, for instance by asking for information, cross-assessment of ideas or mutual monitoring of work progress. It encourages knowledge construction, skill development and deeper understanding by actively engaging people in the learning processes [12].

At the origin of the $3 \mathrm{C}$ collaboration model, originally proposed by Ellis et al [7] and later explored in [6], is the combination of communication, coordination 
and cooperation. Communication is related to the exchange of messages and information among people; coordination is related to the management of people, their activities and resources; and cooperation refers to any kind of production taking place collaboratively, on a shared space. This model appears frequently in the literature as a mean to classify collaborative systems.

In the paper Applying the 3C model to groupware development [11] two instantiations of the $3 \mathrm{C}$ model are presented, as well as the classification of AulaNet services based on the 3C model. The first instantiation is for Mackay's Media Space [13], which are multimedia, enhanced communication spaces. The Media Space itself is the shared space. It is aimed at informal communication and its main goal is to create opportunities for informal meetings, which are coordinated by the standing social protocol. Such meeting generate conversation, which may occur using the media provided by the system or any other available mean, such as telephones. The instantiation is depicted in Figure 1.

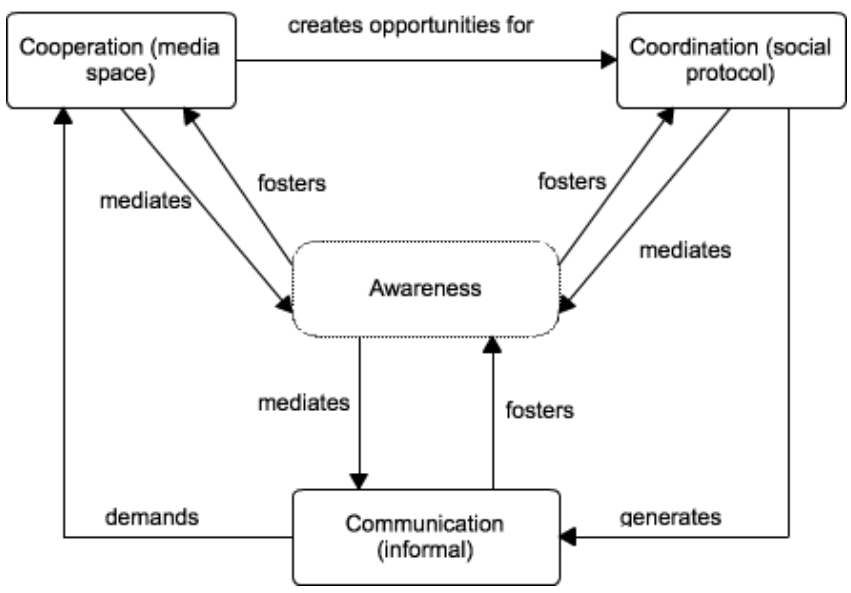

Fig. 1. 3C collaboration model instantiated for the Media Space domain.

A second instantiation is concerned with group work and is depicted in Figure 2. The $3 \mathrm{C}$ collaboration model instantiated to the groupware domain shows that, while communicating, people negotiate and make decisions. While coordinating themselves, they deal with conflicts and organize their activities in a manner that prevents loss of communication and of cooperation efforts. This example shows the iterative nature of collaboration.

Although, as seen above, the 3C model is commonly used for classifying collaborative systems, no attempt was done to use it in the FLOSS development scenario. FLOSS builds on the general idea of open and available source code and goes even further by being both a software development method and a software business model [16]. It is further defined by the license used to grant users and 


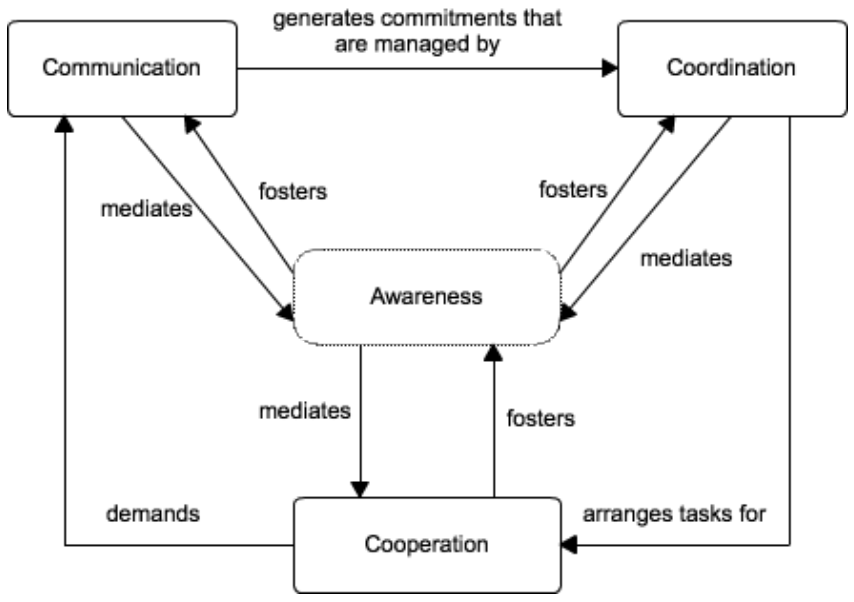

Fig. 2. 3C collaboration model instantiated for group work.

developers additional right to the code. FLOSS can freely be used, studied, and modified. Note that it is not necessarily cost free, thus qualifier free does not relate to monetary cost but to freedom or liberty. Copying and redistribution is allowed, but can be restricted by the license as it may require a need to grant the same rights to the modified versions as well. Richard Stallman formulated the first definition of free software in 1983, as any piece of software that grants anyone with a copy the freedom to run, study, redistribute or improve it. Actually, there are four degrees of freedom used to classify FLOSS [10]. A program is free software if it gives users adequately all of these freedoms. Otherwise, it is non free. Those are indexed from zero as geek homage to zero-based numbering often used in computer systems, as follows:

0 The freedom to run the program for any purpose.

1 The freedom to study how the program works, and change it to make it do what you wish.

2 The freedom to redistribute copies so you can help your neighbour.

3 The freedom to improve the program, and release your improvements (and modified versions in general) to the public, so that the whole community benefits.

At the heart of FLOSS is the developer community a social ecosystem on its own. The structure of the community is often depicted with a layered onion model, where the users of the software form the outmost layer and the most prominent developers and the leader of the project are at the core [15]. As depicted in Figure 3, the Onion Community Model for FLOSS focuses on the developer community alone. 


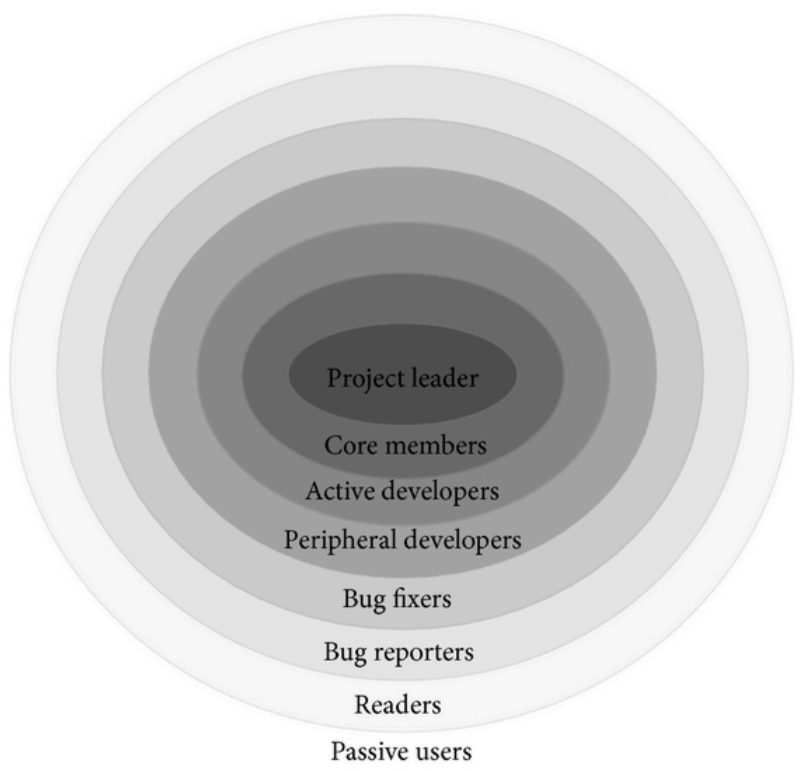

Fig. 3. General Structure of a FLOSS Community (Onion Model).

\section{$3 \quad$ Research Method}

The aim of this study was to understand how collaboration and learning occurs within FLOSS communities; which are the motivations, challenges and difficulties participants in such projects experience, as well as which sort of learning experience they have and how they interact. Research was based on qualitative instruments, as described below. Actually, qualitative research methods produces results that cannot easily be achieved by statistical procedures or similar quantitative methods [18]. The results of this kind of approach are richer and more informative, helping to answer questions involving variables that are difficult to quantify, such as human characteristics like motivations or perceptions.

For this study we used both a survey and structured interviews as data collection method. The survey was made available online using Google Docs and shared among FLOSS communities, as well as sent to a number of individual FLOSS contributors. Its aim was twofold 1) to study the interactions between FLOSS projects participants, and 2) to assess the didactical value of participating in FLOSS projects. A preliminary analysis of this survey was previously made in references $[8,9]$. The interviews were later conducted to a number of FLOSS contributors selected among those who have previously replied to the online survey.

The questionnaire was structured into three main sections: 
- Section A collects respondents demographics, including age, country, language, background and the different FLOSS projects he/she have been enrolled in.

- Section B collects data about the respondents interaction with the project community, and the motivations to start and continue contributing to FLOSS project. It explores the respondents participation in a specific FLOSS project. The respondent is faced with a specific project, to which he/she has (or is) contributing, and is requested to describe how the participation started, the drivers what drove him/her to starting such activity, his/her role in the project, and how many hours he/she devotes to it. The respondent has the opportunity to describe the type of relationships he has with other community members, how they share information, or if they promote and participate in community meetings or events.

- Section C surveys where the respondents exploit the potential of FLOSS projects as learning environments. The respondent is asked whether the fact of being in a FLOSS community provides him with a learning opportunity, and if his background (professional or academic) facilitates the learning process while participating in a FLOSS project. The identification of the most important agents in this learning process is also addressed, as well as if FLOSS projects can be regarded as 1) learning communities, 2) a possible alternative to formal education, and 3) an interesting complement to formal education.

Each section comprises open-ended and closed-ended questions. In the openended questions no possible responses were given, allowing the respondent to write down the answers in his/her own words. In the closed-ended questions, possible answers were provided for the respondent to tick the category that best described his/her choice. In such questions, the clause "Other / please explain" was included to accommodate any response not listed. The use of these two forms of questions revert to the fact that close-ended questions are extremely useful for eliciting factual information and open-ended questions for seeking opinions, attitudes and perceptions. In the closed-ended questions, we not only allowed multi-selection answers but also provided three types of Likert Scale answers: 1) to analyze the respondents perception, including values like Strongly disagree, Disagree, Not sure/ Not applicable, Agree, and Strongly agree; and 2) to analyze the frequency of certain respondents behavior, including values like Ever, Once every year, Once a month, At least 3 times per month, and More than 3 times per month; and 3) to assess the relevance that the respondent assigns to a specific issue, including values like Not at all important, Not too important, Not sure / Not applicable, Somewhat important, and Very important.

The interviews, used in a second phase of this study, aimed at further exploring 1) the motivations to participate in FLOSS projects, and 2) the didactical value of their communities (emphasising namely, the interactions established while contributing to the project or carrying on related activities. 


\section{Data Analysis}

Questionnaire Results. Since the questionnaire was released, data was collected from 28 respondents, 25 men, 3 women, from 16 different countries, including Portugal, United Kingdom, Germany, India, France, Serbia, Finland, Netherlands, Belgium, Slovenia, USA, Macau SAR China, Canada, Argentina, Israel, and Brazil (see [9] for a detailed analysis).

Concerning the first question-objective, we were able to see that, independently of the type of relationships participant maintain with FLOSS communities and the degree of personal acquaintance, such communities act as important personal networks, promoting high-level interactions and creating opportunities to (often virtually) meet and socialize in a variety of events. Although the most commonly given reason for starting contributing to a FLOSS contributors project, was the response to a personal/intellectually rewarding challenge, their interest does not fade out along time and they go on contributing to the project in a quite regular way.

Concerning the second research question, it became evident that FLOSS projects contributors recognize the learning potential of such environments, and that the fact of being active participaticipants in such projects improves a number of different skills. All the respondents identified not only other community members as learning agents, but also include themselves in such a category. This provides evidence of the eminently collaborative natures of the, often non linear but effective teaching and learning processes occurring within FLOSS.

Interviews to FLOSS contributers. After conducting the questionnaire online, a group of 4 participants from the survey was selected to be interviewed individually. The data collected is summarised and briefly analysed below. As already mentioned, the focus was put on the motivations to participate in FLOSS projects and communities, on the one hand, and the assessment of such an experience as a possible learning one, on the other.

Interview 1. The first FLOSS developer to be interviewed, referred as interviewed $\mathbf{A}$ in the sequel, was a master student, who acts as a freelance software consultant and developer. For a long time, he has been engaged in several FLOSS projects, such as cwac-caera, UniversallmageLoader, ProgreeWheel and Django. For the purpose of the interview A chose to refer to the cwac-camera project.

A decided to participate in the cwac-camera project after using this software at a professional level. In his case, the interactions with the community were easy and the communication was exclusively done online, via Github, resorting to issues/forums or pull-requests. He communicates weekly with other community members and is willing to help whoever seeks support. The interviewed never had any serious disagreement with community members nor he motived others to join the community. For him, the time of participation on such projects depends only on the personal interest and time available, as the projects can only achieve the most if contributors are motivated and driven to move it forward. 
With respect to the possible learning experience developed along the process, A believes he has learned a lot, particularly on how others contribute to the same project and how they interact among each other and with code being produced. Moreover, as he is willing to help others, he also likes to share his knowledge.

When asked if participating in FLOSS project in the the context of formal education, A believes that everyone studying software development and engineering should at least once experience what it means to participate and contribute to a FLOSS project. This is understood as a privileged way to learn and get proficient in a number of methods, techniques and professional practices, as well as in purely social skills. As a limitation to use FLOSS projects in formal education, A points out the control students may or not have of the project and its size. Those are factors that may limit how broad or how deep the learning experience may result. He also mentions that the success or failure of the particular project used in such a context cannot be used as an reliable instrument for assessing one's contribution.

Interview 2. The second interview was made to FLOSS contributer B. He holds a $\mathrm{PhD}$ and is professinaly an university lecturer. $\mathbf{B}$ has been involved with several FLOSS projects, such as NetBSD, EDOS/MANCOOSI, Coq, or FreeBSD. For the purpose of the interview, $\mathbf{B}$ selected the NetBSD project.

B decided to participate in NetBSD project because he felt he could contribute with some patches and, moreover, there were some bugs that he knew how to fix. He communicates with other community members once a week through the project mailing lists. Despite his wish, his participation is somehow limited due to lack of time. Since B joined the community, a very opinionated one, he became aware of several on-going disagreements and quarrels inside it, but tries to stay away from them, depending on the points being discussed. For $\mathbf{B}$, both a long term or a short term period of participation in a project can make sense, both of them bringing a number of advantages. Actually, in hos perspective, FLOSS projects should allow both short-term participations for submitting occasional patches (e.g. to fix a bug) and long-term development to mature ideas and practices and bring a sense of continuity to the project. As far as difficulties are concerned, $\mathbf{B}$ claims they are inevitable, but underlines the support always offered by other community members.

As for the learning experience, $\mathbf{B}$ recognises he indeed learned some new technical skills and new ways of interacting with other developers. Facing people with strong personalities and commitment, lead to the development of new and improves socially-related skills.

When asked about the possible use of participation in FLOSS projects as an element in formal education, $\mathbf{B}$ believes it represents a great opportunity to interact with the FLOSS world and with a remarkable formative value. He is promoting a Software Engineering course at his university that uses FLOSS projects as part of the syllabus. 
Interview 3. The third interview, referred as $\mathbf{C}$ in the sequel, also has a $\mathrm{PhD}$. $\mathbf{C}$ has been involved with several FLOSS projects, such as Parrot VM, Rakudo, Perl, and Dancer.

For the purpose of this interview, $\mathbf{C}$ selected the project Dancer, a lightweight web framework written in Perl for building websites and similar applications. C started participating in the project as, by that time, he was already involved in building application, and decided to help on a new version. $\mathbf{C}$ communicates in a daily basis with the community, using IRC, mailing lists or the issue tracker available.

The Dance community is very active, and without having much time, $\mathbf{C}$ have difficulties and keeping up with everything happening, there. However, he tries to remain open to challenges and new problems to solve.

The community does not have disagreements but does have "healthy discussions. The motivation and the time spent in the project, for $\mathbf{C}$, depends on personal interest and the fact that when a difficulty arises, community members are always willing to help.

As for the learning experience, $\mathbf{C}$ believes participating in FLOSS projects constitutes a source of learning, of new skills and technologies, as many people with different backgrounds and different education paths share their knowledge. What one learns and the knowledge created is, for $\mathbf{C}$, the best benefit one can have for participating in a FLOSS project. Also, $\mathbf{C}$ believes that FLOSS projects should be used as learning experiences and that the active involvement in such communities should be formally included in some courses of at university level.

Interview 4. The fourth interview was with interviewed $\mathbf{D}$, a university lecturer, that has been contributing to the Perl community. $\mathbf{D}$ does not contribute to a single project but is involved in a set of modules, each of which can be regarded as a project in itself.

D was motivated to participate in such project because he was an enthusiast of the language; but also because he got to know someone of the community. He began the interaction via email, with other community members, asking for help to use some of the modules. Now they use IRC channel on a regular basis.

D is motivated by the project mainly because he enjoys seeing the community growing, with a general and active participation. Actually, there are almost no cases of people joining and dropping out of the project in the first month. As far as the time of participation is concerned, only those with more time in the community can advance for the development of more complex projects (modules). Hence, it depends on what is the task to perform. The community is very helpful and every time $\mathbf{D}$ had a difficulty he found the solution with a community member. Similarly he is willing to help others, a dynamics that seems to be dominant in this sort of communities.

Concerning the learning experience, $\mathbf{D}$ acknowledges to have learned many different things and progressing in different aspects. For example, he learned how to deal with other people or even to improve the use of English. He also became aware of how people work differently, how to read, analyse and reuse 
other peoples code and deal with new technologies from a very practical starting point.

For D using FLOSS project as a learning platform at different level of education can be beneficial but also represent a risk. Students may lack the basic knowledge he believes to be required, or, on the other hand, FLOSS communities may not have the patience or time to help them. This may be purely out of their horizon. His recommendation is to proceed in a careful way, as there are no two students or two FLOSS communities equal.

Summary of findings. The data gathered both through the questionnaires and the four interviews to FLOSS developers provide empirical evidence on

- The relevance as well as the complexity of the human interactions involved; remarkably in all interviews FLOSS communities were mentioned as a sort of school for personal relationships.

- The strongly personal motivation for joining and remaining such communities.

- The mutually suportive environment.

- The intense, even if sometimes unbalanced, learning experience offered. Most, but not all, see in participation in such projects an opportunity that could be somehow integrated in a formal education setting.

These findings were taken into consideration in our proposal of a $3 \mathrm{C}$ collaborative model for FLOSS communities, detailed in the next section.

\section{A 3C Collaborative Model for FLOSS Communities}

As referred in Section 1, the origin of the $3 \mathrm{C}$ collaboration model is the combination of communication, coordination and cooperation. FLOSS communities are all about collaboration, as witnessed by the interviews summarised above. They grow because people gather in the same goal: to develop software. To do it, collaboration is at the core of its success, despite personal motivations, backgrounds or professional activities. However, the outcome of the FLOSS community dynamics is well known and not only such project are often successful in terms of products made available (with a growing commercial impact, it should be noted), but also in terms of the knowledge produced and the communities fostered along the process. Indeed all participants, in different ways, have the opportunity to learn both new technical skill, but also new social and cultural skills, new ways of working, etc. Hence FLOSS community can accurately be described in terms of the $3 \mathrm{C}$ collaborative model proposed by [7].

Figure 4 depicts our proposal to instantiate a $3 \mathrm{C}$ collaborative model applied to FLOSS communities. As previously observed, motivation is the key to start participating in a FLOSS project; it can be a new challenge, the topic, a new idea, etc. The tasks developed are just one of the ways to contribute. FLOSS projects are developed within a communitarian basis. The knowledge each participant brings can help not only the project to succeed, but also others 


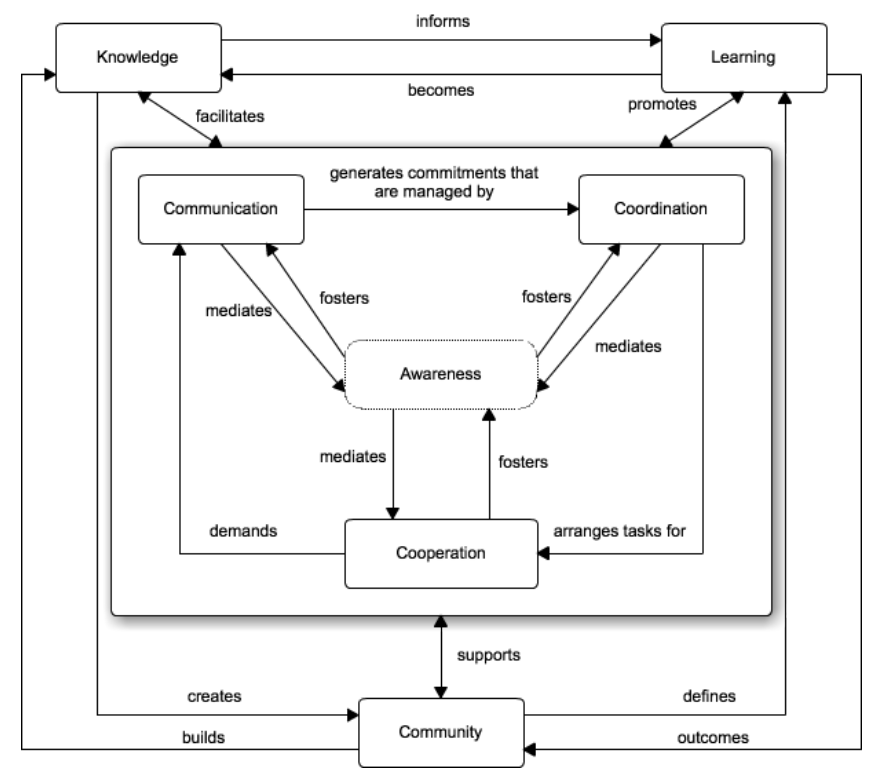

Fig. 4. The 3C Collaborative model instantiated for FLOSS Communities.

in acquiring new knowledge and skills. As observed in the interviews, new knowledge can be acquired when a FLOSS participant faces difficulties, or sometimes it happens just out of curiosity and personal motivation. Why knowledge happens is of minor importance, in comparison with how knowledge is facilitated and how learning is promoted within a FLOSS community. This instantiation of the $3 \mathrm{C}$ collaboration model for FLOSS communities allows for the description of their dynamics which can be regarded as a learning experiences, with relevant didactical potential.

\section{Conclusions and Future Work}

FLOSS communities consist of heterogeneous groups of independent volunteers who interact among them driven by different motivations, to produce a shared software asset. As we were able to analyze by our sample of respondents to the questionnaire, FLOSS projects participants collaborate and cooperate between them in more systematic and innovative ways than usual in normal, classic professional practice. Al the community dynamics is focused and driven by its shared objective: to develop a new software project. Despite the relevance they give to FLOSS development, it is interesting to see that the FLOSS developers interviewed are skeptical with respect to the possibility that participation in FLOSS projects can be an alternative to formal education, for example, to replace formal courses in Software Engineering in higher education institutions. However, they 
see that the learning by doing concept, typically emerging from FLOSS projects, are an effective and possibly attractive complement to formal education, mainly in Software Engineering degrees. Clearly, the 3C collaboration model can be applied to model FLOSS communities collaborative learning frameworks and help to categorise and assess them.

We are currently working on validating this model in the educational context, through the analysis of data gathered in a pilot project in which MScr students have to join an contribute to a FLOSS project as part of a formal course in Software Engineering.

Aknowlegdments. This work was supported by the Programa Operacional da Região Norte, NORTE2020, in the context of project NORTE-01-0145-FEDER-000037. The second author is further supported by FCT under grant SFRH/BSAB/113890/2015.

\section{References}

1. J. Arets and V. Heijnen. Interview with jay cross (retrieved may 9, 2016). www.tulser. com/wp-content/uploads/2015/10/Tulser-interview-Jay-Cross.pdf, 2015.

2. S. Bacon and T. Dillon. The potential of open source approaches for education. Futurelab, 44, 2006.

3. L. J. Burnell, J. W. Priest, and J. B. Durrett. Teaching distributed multidisciplinary software development. IEEE Software, 19(5):86-93, 2002.

4. Antonio Cerone and Sulayman K. Sowe. Using free/libre open source software projects as e-learning tools. ECEASST, 33, 2010.

5. M. Driscoll. Psychology of Learning Instruction (3rd ed.). Pearson Education, Inc., 2005.

6. Clarence Ellis and Jacques Wainer. A conceptual model of groupware. In Proceedings of the 1994 ACM Conference on Computer Supported Cooperative Work, CSCW '94, pages 79-88, New York, NY, USA, 1994. ACM.

7. Clarence A. Ellis, Simon J. Gibbs, and Gail Rein. Groupware: Some issues and experiences. Commun. ACM, 34(1):39-58, January 1991.

8. Sara Fernandes, Antonio Cerone, and Luís Soares Barbosa. A preliminary analysis of learning awareness in FLOSS projects. In Antonio Cerone, Donatella Persico, Sara Fernandes, Alexeis Garcia-Perez, Panagiotis Katsaros, Siraj Ahmed Shaikh, and Ioannis Stamelos, editors, Information Technology and Open Source: Applications for Education, Innovation, and Sustainability - SEFM 2012 Satellite Events, InSuEdu, MoKMaDS, and OpenCert, Thessaloniki, Greece, October 1-2, 2012, Revised Selected Papers, volume 7991 of Lecture Notes in Computer Science, pages 133-139. Springer, 2012.

9. Sara Fernandes, Antonio Cerone, and Luís Soares Barbosa. Analysis of FLOSS communities as learning contexts. In Steve Counsell and Manuel Núñez, editors, Software Engineering and Formal Methods - SEFM 2013 Collocated Workshops: BEAT2, WS-FMDS, FM-RAIL-Bok, MoKMaSD, and OpenCert, Madrid, Spain, September 23-24, 2013, Revised Selected Papers, volume 8368 of Lecture Notes in Computer Science, pages 405-416. Springer, 2013.

10. GNU Foundation. What is free software? (retrieved may 9, 2016). www.gnu.org/philosophy/free-sw.en.html, 2016. 
11. H. Fuks, A. B. Raposo, M. A. Gerosa, and C. J. P. Lucena. Applying the 3c model to groupware development. International Journal of Cooperative Information Systems, 14(02n03):299-328, 2005.

12. Carlos A. Jara, Francisco A. Candelas, Fernando Torres, Sebastian Dormido, Francisco Esquembre, and Oscar Reinoso. Real-time collaboration of virtual laboratories through the internet. Computers \& Education, 52(1):126 - 140, 2009.

13. Wendy E. Mackay. Media spaces: Environments for informal multimedia interaction. In M. Beaudouin-Lafon, editor, Computer-Supported Cooperative Work, Trends in Software Series, pages 55-82. Wiley and Sons, 1999.

14. P. Magrassi. Free and open-source software is not an emerging property but rather the result of studied design. CoRR, abs/1012.5625, 2010.

15. Kumiyo Nakakoji, Yasuhiro Yamamoto, Yoshiyuki Nishinaka, Kouichi Kishida, and Yunwen Ye. Evolution patterns of open-source software systems and communities. In Proceedings of the International Workshop on Principles of Software Evolution, IWPSE '02, pages 76-85, New York, NY, USA, 2002. ACM.

16. E. S. Raymond. The Cathedral and the Bazaar. O'Reilly Media, 1999.

17. Sulayman K. Sowe, Antonio Cerone, and Dimitrios Settas. An empirical study of FOSS developers patterns of contribution: Challenges for data linkage and analysis. Sci. Comput. Program., 91:249-265, 2014.

18. A. Strauss and J. M. Corbin. Basics of Qualitative Research: Techniques and Procedures for Developing Grounded Theory (2nd ed.). Sage Publications, Inc., 1998.

19. Han-Yu Sung and Gwo-Jen Hwang. A collaborative game-based learning approach to improving students' learning performance in science courses. Computers 8 Education, 63(1):43-51, 2013. 